\title{
Visual evoked potentials in NIDDM: a longitudinal study
}

\author{
G. Moreo ${ }^{1}$, E. Mariani² ${ }^{2}$, G. Pizzamiglio ${ }^{1}$, G. B. Colucci ${ }^{1}$ \\ ${ }^{1}$ Divisione di Medicina Generale, Istituti Clinici di Perfezionamento, Milan, Italy \\ ${ }^{2}$ Servizio di Neurofisiologia, Istituti Clinici di Perfezionamento, Milan, Italy
}

\begin{abstract}
Summary In order to assess the possible progression of neurological abnormalities over time and the value of visual evoked potential alterations in predicting stability and severity of diabetes-related optic pathway disease, a longitudinal study in non-insulin-dependent diabetic patients was performed. Neurological examination, visual evoked potentials with pattern reversal, motor and sensory nerve conduction velocities and metabolic control were studied in 18 non-insulin-dependent diabetic patients and in 35 normal control subjects at baseline and again after $4.6 \pm 0.8$ years (range $4-6$ ). At the first recording the peak $\mathbf{P} 100$ wave latencies were significantly delayed in the diabetic patients compared with the control subjects; signs of peripheral neuropathy were detected in five patients, clinical in
\end{abstract}

three and in two there was only neurophysiological alteration without clinical signs. The second recording revealed no significant alterations of P100 latencies in patients compared with baseline, but the number with clinical signs and/or neurophysiological alterations with no clinical signs of peripheral neurological disease was increased to seven. In conclusion, we observed that visual evoked potential alterations were stable over time whereas peripheral neurological disease progressed and correlated positively with metabolic control. [Diabetologia (1995) 38: 573-576]

Key words Non-insulin-dependent diabetes mellitus, visual evoked potentials, diabetic neuropathy, optic pathways, diabetic complications.
The alterations of visual evoked potentials in diabetes are now well-known. Most studies agree on the sensitivity of visual evoked potentials with pattern reversal (VEP-PR) in detecting involvement of the optic pathways at the preclinical stage in both diabetic adults [1$3]$ and children $[4,5]$. Recently we also observed VEPPR alterations in diabetic patients without retinopathy and showed a correlation with diabetic polyneuropathy [6]. The predictive significance of such altera-

Received: 6 June 1994 and in revised form: 27 September 1994

Corresponding author: Dr. G. Moreo, Divisione di Medicina Generale, Istituti Clinici di Perfezionamento, Via S. Barnaba, 8, I-20122 Milan, Italy

Abbreviations: VEP-PR, Visual evoked potentials with pattern reversal; NCV, nerve conduction velocities; LP100, peak latencies of P100 wave; ILD, interocular latencies difference; NIDDM, non-insulin-dependent diabetes mellitus; ARI, aldose reductase inhibitor. tions, particularly if detected at the preclinical stage, has been studied less. We therefore performed a longitudinal evaluation of VEP-PR as well as clinical signs and neurophysiological alterations without clinical signs of peripheral neurological disease in a homogeneous population of subjects with non-insulin-dependent diabetes mellitus (NIDDM) without retinopathy and with normal visual acuity at baseline. The aim was to assess the possible progression of neurological abnormalities with time and the value of VEP-PR alterations in predicting the stability and severity of optic pathway disease related to diabetes.

\section{Subjects, materials and methods}

Subjects. We studied VEP-PR, peripheral nerve conduction velocities (NCV) in three nerves, and metabolic control in 18 diabetic patients without retinopathy and in 35 normal subjects. After at least 4 years $(4.6 \pm 0.8$ years, range $4-6)$ a sec- 
Table 1. Mean amplitude (A) VEP latency (P100) and interocular latency difference (ILD) in diabetic patients and control subjects at baseline and follow-up

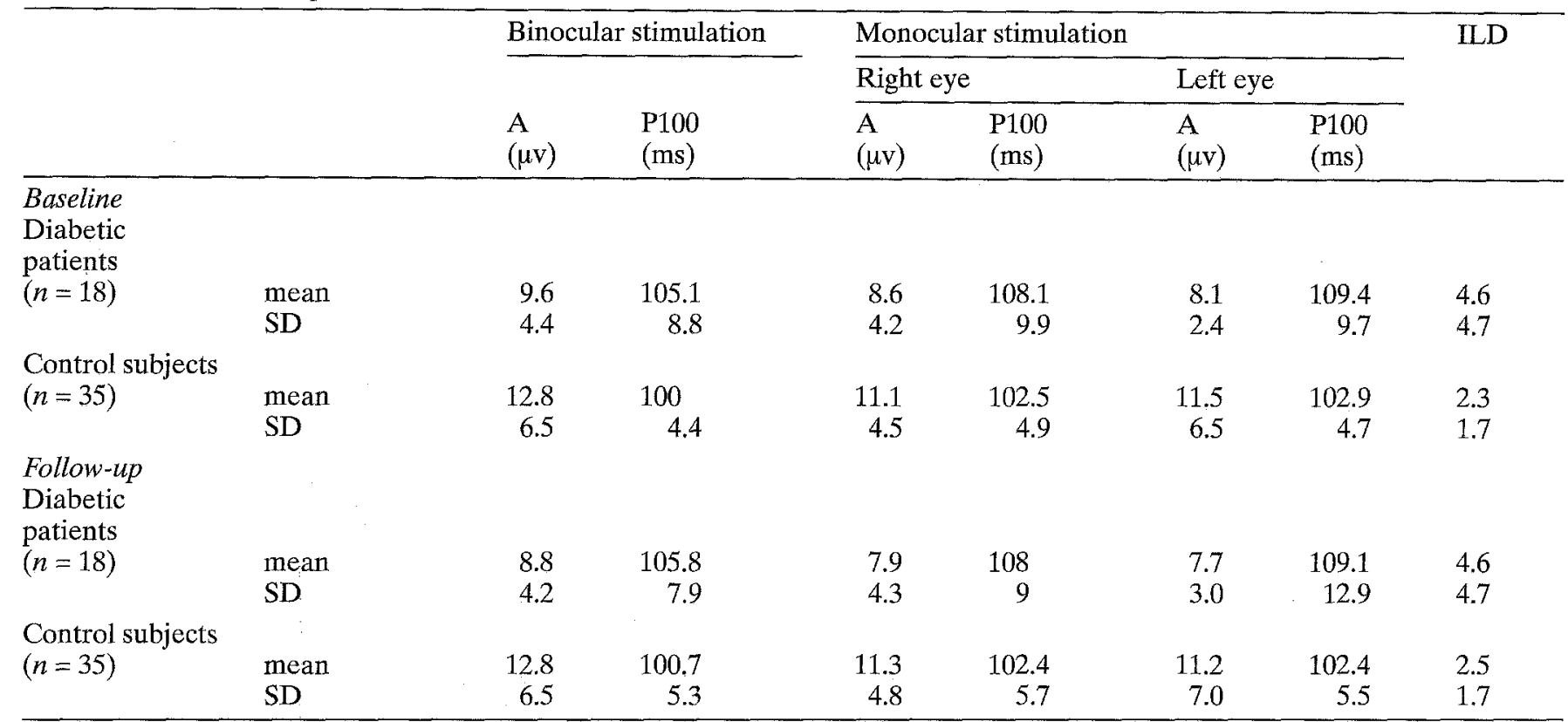

Patients vs control subjects at both baseline and follow-up: NS (amplitude), $p<0.002$ (P100 binocular and P100 right), $p<0.02$ (P100 left and ILD)

ond identical study was carried out in all the patients and in the normal control subjects by the same personnel as in the baseline study. The diabetic group consisted of 11 men and 7 women, all non-insulin-dependent according to the National Diabetes Data Group [7]; at baseline their mean age was $51.1 \pm 6$ years, with mean duration of disease since diagnosis of $7.9 \pm 5.3$ years (range 1-23), and they were all treated with oral hypoglycaemic agents. The patients were also subdivided into two groups according to the presence or absence of clinical signs and/or neurophysiological alterations without clinical signs of peripheral neurological disease; the presence of the latter was considered if there were alterations of at least two of the four NCV values. The control group consisted of 35 healthy subjects $(20$ men, 15 women, mean age $49 \pm 7.3$ years at baseline) without a family history of diabetes mellitus; it was strictly the same at the second recording. All subjects in the study had a negative history and negative physical examination for central nervous system disease, normal ophthalmologic examination, and normal visual acuity with or without correction. Inclusion criteria in the diabetic subjects were normal electroretinogram and fluorangiography at baseline. Exclusion criteria were alcohol consumption above the equivalent of $500 \mathrm{ml}$ wine per day for men and $350 \mathrm{ml}$ for women, cigarette consumption of over ten cigarettes per day, and the presence of peripheral nervous system disease unrelated to diabetes. Prior to participation the purpose of the study was explained to all of the patients and control subjects and their informed consent was obtained.

Laboratory methods, visual evoked potentials and nerve conduction velocities. Metabolic control was evaluated by the mean level of $\mathrm{HbA}_{1 \mathrm{c}} \%$ measured in a sample of venous blood and determined by HPLC using ion-exchange columnchromatography (normal values 3.5-6.4\%). The $\mathrm{HbA}_{1 \mathrm{c}}$ level at baseline was measured on the day of the first VEP recording. The $\mathrm{HbA}_{1 \mathrm{c}}$ level of the second recording corresponded to the mean of 12 values obtained from each subject in tests performed once every 3 months during the last 3 years of follow-up. VEP-PR were always recorded with the same equipment using binocular and monocular stimulation according to the standardized protocol proposed by Chatrian [8]. Stimulation was performed with a $25 \times 18 \mathrm{~cm}$ screen with black and white checker-board pattern, each square with $1.1 \mathrm{~cm}$ sides. The screen was placed $1 \mathrm{~m}$ from the nasion (corresponding visual angle, $38^{\circ}$ ), with the aim of stimulating the foveal and parafoveal fibers at $75 \%$ contrast and reversal rate of $1 \mathrm{~Hz}$ under conditions of constant brightness. The response was evaluated by active electrodes placed over the occipital region at $\mathrm{O}_{2}(5 \mathrm{~cm}$ above the inion $), \mathrm{O}_{1}$ and $\mathrm{O}_{2}(5 \mathrm{~cm}$ lateral to the midline electrode, at the left and at the right respectively) and a reference electrode placed at Fz. A total of 128 tests, repeated once, were analysed, and responses showing excessive artifacts were excluded. The system bandpass was between 0.5 and $1000 \mathrm{~Hz}$ and the analysis time $200 \mathrm{~ms}$. We measured the peak latencies of P100 wave (LP100), the amplitude of the potentials (calculated from the N75 peak to the $\mathrm{P} 100$ peak in $\mathrm{Oz}$ ) and the interocular P100 latencies difference (ILD) at monocular stimulation. LP100 higher than 3 $\mathrm{SD}$ of the mean of the control subjects were considered pathological according to Chiappa [9]. Four standard NCV (motor and sensory NCV of the median nerve, motor NCV of the peroneal nerve, sensory NCV of the sural nerve) were determined in all the diabetic patients according to Kimura's methods and criteria of interpretation [10] using Alvar Myodyne PEM II equipment (Montreuil, Paris, France) and they were considered pathological when they were less than $2 \mathrm{SD}$ of the mean of the control subjects.

Scores were calculated for each variable (NCV, VEP-PR, $\mathrm{HbA}_{1 \mathrm{c}}$ ) based on the comparison of alterations between the first and second recording as follows: NCV score from -4 to +4 , VEP-PR score from -3 to +3 , and $\mathrm{HbA}_{1 \mathrm{c}}$ score from -2 to +2 , where negative signs indicate improvement and positive signs indicate worsening of at least 2 SD (i.e. VEP-PR score of -2 indicates improvement of two of the three VEP- 
$\mathrm{PR}$ recorded at follow-up). For $\mathrm{HbA}_{1 \mathrm{c}}$ a score of 1 indicated a variation of at least $30 \%$ and 2 a variation of at least $60 \%$.

\section{Statistical analysis}

Statistical analysis was performed using Student's $t$-test and correlations were evaluated by calculations of the coefficient $r$. The results are expressed as mean \pm SD. Values of $p<0.05$ were considered significant.

\section{Results}

At baseline LP100 were significantly increased at both binocular and monocular stimulation in the 18 diabetic patients compared with the control subjects, as well as ILD between diabetic patients and control subjects (Table 1 ). Seven of the 18 patients $(38.8 \%)$ had LP100 greater than 2 SD and five $(27.7 \%)$ greater than $3 \mathrm{SD}$ of the mean of the control subjects. At the second recording, performed at least 4 years later, the LP100 and the ILD of the patients were still increased compared with the control subjects and the differences were highly significant (Table 1). On the other hand, the 18 diabetic subjects showed mean LP100 values which were very similar to those at baseline and the difference between the first and second recording was minimal: nine had identical values, four a mild decrease ( $>1$ SD) of LP100 in one of the monocular recordings and one only in the binocular recording, and four a moderate increase $(>1$ SD) of LP100 in at least one monocular recording. Also ILD was unchanged (Table 1). Moreover, when we analysed polyneuropathy in the diabetic patients we observed a tendency to progression of peripheral nervous system involvement. Signs of peripheral neurological disease were seen in five patients at baseline, clinical in three and only neurophysiological alteration without clinical signs in two, whereas at the second recording clinical signs were confirmed in three subjects who had clinical signs at baseline and detected in two with only neurophysiological alterations without clinical signs at baseline; the latter were seen newly developed in two other subjects.

Five patients presented initial background retinopathy at the follow-up examination, without altered visual acuity, and only one of them had a mild increase in left monocular and binocular recordings at follow-up compared to baseline.

Diabetic patients were in good metabolic control at baseline as well as during the period of follow-up; mean $\mathrm{HbA}_{1 \mathrm{c}}$ was $6.66 \pm 1.17 \%$ at the first recording and $7.54 \pm 1.3$ at the second.

Correlations were sought between VEP-PR score, $\mathrm{NCV}$ score, and $\mathrm{HbA}_{1 \mathrm{c}}$ score, and a positive significant correlation was found between the NCV and $\mathrm{HbA}_{1 \mathrm{c}}$ scores $(r=0.64, p<0.01)$.

\section{Discussion}

In line with other authors' $[1,3,11,12]$ and our previous findings [6], differences in VEP-PR were observed between diabetic patients and control subjects in the present longitudinal study. LP100 was significantly increased at both monocular and binocular stimulation in our patients with NIDDM at baseline and after 4 years. Initial background retinopathy, documented in five patients at the follow-up examination, did not seem to influence LP100.

Such alterations of the optic pathways, little-studied in the early stage, as the rare autopsy findings document alterations in very advanced stages only [13], and consisting of severe demyelination and degeneration of the axis cylinders in the optic chiasma, are in line with observations in patients with myelopathy and encephalopathy associated with diabetes $[14,15]$. This suggests that the central nervous system is not spared from involvement in diabetes.

Moreover, in our study VEP-PR abnormalities persisted in all the patients with pathological values at baseline. In fact, VEP-PR alterations were surprisingly stable considering the progression observed, as expected, in clinical signs and neurophysiological alterations without clinical signs of peripheral neurological disease, as already described [16].

In a recent study on animal models Kamijo et al. [17] demonstrated that axonal atrophy and axo-glial dysjunction are the two structural lesions that occur in optic neuropathy, similar to the lesions of diabetic peripheral neuropathy in humans [18]; they are responsible for prolongation of the latency of the optic nerve response and are'probably polyol-pathway dependent. On the other hand, these authors also demonstrated that the lesion of the central sensory nerve tract is less severe, and that axo-glial dysjunction is completely prevented by treatment with an aldose reductase inhibitor (ARI) whereas axonal atrophy is not. This is in contrast to the situation in the peripheral nerve, where ARI has a significant preventive effect on axonal atrophy [19].

The prolongation of LP100 which we observed is thus an expression of structural damage at the level of the myelinated optic nerve fibers, which is closely related to axo-glial dysjunction, and the differing progression of neurological abnormalities in the peripheral nervous system and optic pathways may suggest a different pathogenesis, or a similar pathogenesis with a different clinical course. The latter hypothesis is supported by the important anatomic differences between the peripheral nervous system and the optic nerve, which has a similar structure to that of the central nervous system [20] and by the finding that metabolic control in our patients in the previous 3 years correlated positively with NCV modifications but not with VEP-PR. A certain reduction in the amplitudes of the visual evoked potentials in diabetic sub- 
jects was observed at both baseline and follow-up, but statistical significance was not reached. This is in line with the findings of Kamijo et al. [17], and also with Halliday's observations [21] on demyelinating disease as the slight decrease detected by us may be a reflection of the degree of conduction block secondary to a greater axonal functional susceptibility.

Although numerous studies have evaluated VEPPR modifications in diabetic subjects as measured on single occasions, as far as we are aware only Pozzessere et al. [22] have published a longitudinal study. Our results obtained in a larger series after a much longer follow-up confirm those of Pozzessere et al., who found that VEP-PR alterations were substantially stable after a mean interval of about 15 months.

In conclusion, VEP-PR alterations in NIDDM are not reversible, are stable, and are probably an expression of structural rather than functional abnormalities, as recently proposed also by other authors $[17$, 23]. Thus, VEP-PR abnormalities represent an interesting sign of subclinical disease, but they still cannot be considered totally predictive of subsequent development of ophthalmologic disease.

Acknowledgements. Part of this study was presented at the 27th Annual Meeting of the European Association for the Study of Diabetes, Dublin, Ireland 1991.

\section{References}

1. Puvanendran K, Devathasan G, Wong PK (1983) Visual evoked responses in diabetes. $\mathrm{J}$ Neurosurg Psychiatry 46:643-647

2. Khardori R, Soler NG, Good DC, Devlesc Howard AB, Broughton D, Walbert J (1986) Brainstem auditory and visual evoked potentials in type 1 (insulin dependent) diabetic patients. Diabetologia 29:362-365

3. Anastasi M, Lauricella M, Giordano C, Galluzzo A (1985) Visual evoked potentials in insulin dependent diabetics. Acta Diabetol Lat 22:343-349

4. Comi G, Martinelli V, Galardi G et al. (1987) Evaluation of central nervous conduction by visual evoked potentials in insulin-dependent diabetic children. Acta Diabetol Lat 24:157-162

5. Cirillo D, Gonfiantini E, De Grandis D, Bongiovanni L, Robert JJ, Pinelli L (1984) Visual evoked potentials in diabetic children and adolescents. Diabetes Care 7:273-275

6. Mariani E, Moreo G, Colucci GB (1990) Study of visual evoked potentials in diabetics without retinopathy: correla- tions with clinical findings and polyneuropathy. Acta Neurol Scand 81:337-340

7. National Diabetes Data Group (1979) Classification and diagnosis of diabetes mellitus and other categories of glucose intolerance. Diabetes 28:1039-1057

8. Chatrian GE (1984) Guidelines for clinical evoked potentials studies. J Clin Neurophysiol 1:3-53

9. Chiappa KH (1990) Pattern-shift visual evoked potentials: interpretation. In: Chiappa KH (ed) Evoked potentials in clinical medicine. Raven Press, New York, pp 111-153

10. Kimura J (1985) Electrodiagnosis in disease of nerve and muscle. In: Kimura J, Davies FA (eds) Principles of nerve conduction studies. Saunders, Philadelphia, pp 83-141

11. Pozzessere G, Rizzo PA, Valle E et al.(1988) Early detection of neurological involvement in IDDM and NIDDM. Multinodal evoked potentials versus metabolic control. Diabetes Care 11:473-480

12. Algan M, Ziegler O, Gehin P et al. (1989) Visual evoked potentials in diabetic patients. Diabetes Care 12:227-229

13. Reske-Nielsen E, Lundbaek K, Rafaelsen OJ (1965) Pathological changes in the central and peripheral nervous system of young long-term diabetics. Diabetologia 1: 233241

14. De Jong RN (1977) CNS manifestations in diabetes mellitus. Postgrad Med 61:101-107

15. Skenazy JA, Bigler ED (1984) Neuropsychological findings in diabetes mellitus. J Clin Psychol 40:246-258

16. Dyck PJ, Thomas PK (1993) Diabetic and hypoglycaemic neuropathy. In: Dyck PJ, Thomas PK (eds) Peripheral neuropathy. Saunders, Philadelphia, pp 1219-1241

17. Kamijo M, Cherian PV, Sima AAF (1993) The preventive effect of aldose reductase inhibition on diabetic optic neuropathy in the BB/W-rat. Diabetologia 36:893-898

18. Sima AAF, Nathaniel V, Bril V, McEwen TAJ, Greene DA (1988) Histopathological heterogeneity of neuropathy in insulin-dependent and non-insulin-dependent diabetes, and demonstration of axo-glial dysjunction in human diabetic neuropathy. J Clin Invest 81:349-364

19. Sima AAF, Prashar A, Zhang WX, Chakrabarti S, Greene DA (1990) Preventive effect of long term aldose reductase inhibition (Ponalrestat) on nerve conduction and sural nerve structure in the spontaneously diabetic BB-rat. J Clin Invest 85:1410-1420

20. Hogan MJ, Alvarados JA, Weddel JE (1971) Histology of the human eye. Saunders, Philadelphia

21. Halliday AM (1993) The visual evoked potential in the investigation of diseases of the optic nerve. In: Halliday AM (ed) Evoked potentials in clinical testing. Churchill, Livingstone, pp 195-278

22. Pozzessere G, Rizzo PA, Valle E et al. (1989) A longitudinal study of multimodal evoked potentials in diabetes mellitus. Diabetes Research 10:17-20

23. Martinelli V, Piatti PM, Filippi M et al. (1992) Effects of hyperglycaemia on visual evoked potentials in insulin-dependent diabetic patients. Acta Diabetol 29:34-37 